Send your letters to the Editor,

British Dental Journal,

64 Wimpole Street

London

W1G 8YS

Email bdj@bda.org

Priority will be given to letters less than 500 words long.

Authors must sign the letter, which

may be edited for reasons of space.

\section{A LEGISLATIVE REQUIREMENT}

Sir, I should like to respond to the letter HTM 01-05 revision (BDJ 2009; 207: 144-145).

I am very concerned that your correspondent is advocating not following guidance, especially as it is a legislative requirement for those with an NHS contract to follow latest guidance. Where fitness to practise issues are raised with the GDC, compliance with current guidance would be relevant, whilst always acting in the patients' best interests.

When primary care dental providers are required to register with the Care Quality Commission, they will need to comply with the Health and Social Care Act 2008 Code of Practice for the NHS on the prevention and control of Healthcare Associated Infections and related guidance (HCAI Code of Practice). The HTM 01-05 is referenced in the revised Code.

The author is correct in thinking that an emphasis in developing this guidance was on protein removal through washing/disinfection. Recent studies from the Health Protection Agency commissioned by the Department have indicated that the use of a washer-disinfector reduces the range of residual general biological and protein contamination on instruments: in particular, the very high levels of contamination seen in less well-controlled methods are absent when validated washer-disinfectors are applied correctly. If we can make all washer/disinfectors as efficient as the current best this should produce an improvement, which would almost eliminate the prion risk from low and most medium risk tissue contact. This would be very effective in the dental situation.

The products to which Dr Kilcoyne refers were well known to the Stake- holder Advisory Group when it was producing the guidance. We have a report on prion deactivation techniques from the Engineering and Science Advisory Committee, and a small number of these products have also been put to the Rapid Review Panel by commercial manufacturers. None of these products has met the criteria for the highest grading, partly because of the need to provide the means of incorporation into the decontamination cycle in such a way that a sterilised product results.

The use of these products at the moment would not substitute for any part of the existing cycle of cleaning and sterilisation, but would add a further stage which would involve a half hour soaking in an enzymally active hot caustic liquid.

We would wish to avoid hazards derived from baths of caustic liquids, particularly if decontamination has to be carried out in the same room as is used for clinical care. Under existing essential quality requirements, COSHH regulations would apply and significant precautions would be required.

It is interesting to note that the first line in the 'instructions for use' section for the prionzyme product information sheet requires the operator to 'follow all existing guidelines (national and institutional) and practices for safe handling of soiled and contaminated instruments.'

The HTM guidance is not a move towards hospital style sterilisation processes; it is specific to local practice based decontamination procedures and has many differences from the draft acute sector guidance reflecting the different needs and infection transmission risks in primary dental care.
The cycles presently used by small washer disinfectors are designed to remove a wide range of biological contaminants and the range of proteins ordinarily present on surgical instruments. The evidence suggests that hydrophobic proteins (including prion proteins) will not be bound by the hydrophilic molecules present. The Department is currently engaged in research to optimise the removal of hydrophobic proteins from dental instruments in washer-disinfectors.

The guidance is a living document and although hard copies will be posted out to individual practitioners as soon as possible, we will also update the electronic version and provide this information to dentists as new evidence emerges. Indeed, the Dental National Decontamination Survey taking place at present will provide evidence for future revisions to this guidance.

HTM 01-05 is evidence based. Over 60 references to the published scientific and clinical literature were used in its compilation. A list of these references is currently being compiled for publication if required. The exception to this rule are the statements on storage times following instrument wrapping after the use of Type $\mathrm{N}$ and other steam sterilisers. The quality of evidence for the storage timescales selected falls below that usually employed by $\mathrm{DH}$. As a result, further research has been commissioned from University College Hospital, London.

I should be very grateful if you would bring this issue to the attention of your readers as soon as possible.

B. Cockcroft By email DOI: 10.1038/sj.bdj.2009.858 


\section{WATER SWISHING}

Sir, it is good to read that the University of Salford and Unilever Oral Care are collaborating in their public-private partnership to improve oral health across 38 countries (commissioned by the FDI World Dental Federation) through the Live, Learn, Laugh programme. ${ }^{1}$

In addition to brushing teeth, swishing of water after food and soft drinks consumption and between meals can be a safe, economic and easy means to improve oral hygiene in developing countries which is much overlooked. Oral irrigation is an effective alternative to manual tooth brushing and dental floss for reducing bleeding and gingival inflammation. ${ }^{2}$ Dental waterjets and mouthwashes are expensive but vigorous water swishing using movement of the lips, tongue, cheeks can be a beneficial alternative for good oral hygiene.

Swishing 20-30 ml of water after eating food or consumption of soft drinks and also between meals for two to five minutes can be of help in removal of loosened food particles, dead cells and mucus from the oral cavity. ${ }^{3}$

\section{V. Math, P. Balasubramaniam, Mumbai}

1. Focus on world oral health. Br Dent J 2009; 206: 612.

2. Barnes C M, Russell C M, Reinhardt R A, Payne J $B$, Lyle D M. Comparison of irrigation to floss as an adjunct to tooth brushing: effect on bleeding, gingivitis, and supragingival plaque. J Clin Dent 2005; 16: 71-77.

3. Math M V, Balasubramaniam P. Oral health and water. Indian J Nutr Diet 2008; 45: 388-391.

DOI: $10.1038 /$ sj.bdj.2009.859

\section{MRSA INFECTION}

Sir, methicillin-resistant Staphylococcus aureus is no longer only a hospitalbased infection. This infection is now arising from community-based sources, and there are many problems with treatment. ${ }^{1}$ The following case describes an incidence of MRSA dental-cause infection of the submasseteric space arising in the community and discusses the implications of finding MRSA in an orofacial abscess.

A 49-year-old medically well male presented with a three week history of persistent facial swelling following uneventful local anaesthetic extraction of the lower left third molar by his dentist. There was marked trismus which created difficulty with intra-oral examination. An initial temperature was recorded of $38^{\circ} \mathrm{C}$. A diagnosis was made of left submasseteric space infection, with systemic involvement.

Blood results showed an increase in white blood cells to $10.3 \times 10^{9} / \mathrm{L}$, and C-reactive protein level at $65 \mathrm{mg} / \mathrm{L}$, confirming a systemic reaction to infection.

Treatment was instigated immediately with intravenous amoxicillin and metronidazole and IV fluids. The patient underwent incision and drainage of the abscess the following day under general anaesthesia, and a pus sample sent for microbiology. A drain was placed, which was removed two days later and the patient discharged on oral clindamycin and metronidazole.

Three days later microbiology reported a profuse growth of MRSA, sensitive to rifampicin, doxycycline, vancomycin, fusidic acid, gentamicin and resistant to flucloxacillin, erythromycin and trimethoprim. The antibiotic regimen was changed to metronidazole, rifampicin and doxycycline. The patient remained otherwise well, the swelling and trismus gradually resolving.

Methicillin-resistant Staphylococcus aureus (MRSA) is a growing concern around the world. The numbers of staphylococcal-related infections are increasing, as therefore are infection numbers with methicillin-resistant Staphylococcus aureus. ${ }^{2}$ Some Scandinavian countries adopt a 'search and destroy' policy to prevent the spread of MRSA. ${ }^{3}$ Concern among the public is also growing, with many sensationalised and disproportionate claims in the media about this infective cause. Incidences of MRSA infection have been reported in the medical literature since 1961.

MRSA was previously regarded as a hospital-acquired/hospital-based infection. However, MRSA is now proliferating in the community, due to asymptomatic carriers and possibly preventable vector transmission. ${ }^{1}$ Case reports are now emerging of MRSA infections in previously unrecognised situations. Even with new antimicrobial agents, these organisms are still difficult to treat, and carry a mortality rate of $21-23 \%$ when compared with methicillin-sensitive Staphylococcus aureus. ${ }^{4}$
This case highlights the importance of increasing awareness of the probability of MRSA infection in dental-related infections and abscesses even within a community setting. This type of infection has previously been unreported in literature searches.

It also illustrates the renewed importance of requesting culture and sensitivity. This allows adjustment of therapeutic agents to those which are most effective.

\section{K. Valand, P. M. McLoughlin, Dundee}

1. Zetola N, Francis J S, Nuermberger E L et al. Community-acquired meticillin-resistant Staphylococcus aureus: an emerging threat. Lancet Infect Dis 2005; 5: 275-286.

2. Gould F K, Brindle R, Chadwick P R et al. MRSA Working Party of the British Society for Antimicrobial Chemotherapy. Guidelines (2008) for the prophylaxis and treatment of methicillin-resistant Staphylococcus aureus (MRSA) infections in the United Kingdom. British Society for Antimicrobial Chemotherapy. J Antimicrob Chemother 2009; 63: 849-861.

3. Kluytmans J, Struelens M. Meticillin-resistant Staphylococcus aureus in the Hospital. BMJ 2009; 338: 532-537.

4. Cosgrove S E, Sakoulas G, Perencevich E N et al. Comparison of mortality associated with methicillin-resistant and methicillin-susceptible Staphylococcus aureus bacteremia: a meta-analysis. Clin Infect Dis 2003; 36: 53-59.

DOI: $10.1038 /$ sj.bdj.2009.860

\section{CREDIT CRUNCH UDAs}

Sir, the credit crunch seems to be affecting the amount of UDA values offered to performers (associates).

Some newly qualified dentists have been offered as low as £5 per UDA, while those performers who have been working since the new contract took effect in 2006 are seeing their UDA values reduced to a lesser amount due to the credit crunch.

It is my understanding that the Government has taken inflation into account and applies some percentage increase in the contract value every financial year, of which none is passed on to the performers by the principals.

It is becoming quite obvious that this GDS contract favours only the principals and not the performers, even though the performers do most of the hard work. There has been a lack of transparency between principals and performers, with the UDA values passed on to performers not reflecting the UDA values given by the PCTs.

It may be advisable when considering any changes to the GDS contract in the future to take the plight of the 
performers into account. Changes such as setting a reasonable minimum UDA payment for performers and a maximum payment based on experience and qualifications might be made. The PCT on the other hand may decide to have a direct contract with the performers, who in turn would pay the principals the agreed UDA values taking all other expenses into consideration.

This would help boost the morale of performers, especially those who have got student loans and other commitments. It would also prevent crushing the already credit crunched performers!

V. Egemonye, London DOI: 10.1038/sj.bdj.2009.861

\section{FOREIGN INCENSE}

Sir, I would like to share with your readers a very rare case of a radiolucent foreign body in the form of an incense stick used by a small boy to remove food debris from a badly broken down tooth with a wide opening in the crown portion. Incense sticks are widely available in all Indian houses as they are burned after every prayer. These sticks were used for a long period and probably many sticks were broken down in the canal and were pushed and packed in the periapical area. This case highlights the limitations of dental radiology in diagnosing endodontic problems.

A 13-year-old Indian boy was referred to me for extraction of a broken down left central incisor which he said was broken 6-7 years previously. He presented with pain and swelling in connection with that tooth. The tooth was visibly broken down with a large opening on the palatal and incisal side. Considering the age of the patient and aesthetics a root canal treatment followed by a full coverage crown was advised.

The patient was medically fit with no other ailments or illness. Extra-oral examination was unremarkable with no swelling. Intra-orally all permanent first molars were erupted. The deciduous second molars along with the upper deciduous canines were present. The upper left central incisor was fractured with a swelling felt in the labial sulcus just above the apex. The swelling was small and no inflammation was seen in that area. A vitality test was carried out and the tooth was found to be non-vital. There was slight pain on vertical percussion. Considering the age of the patient and the condition of the tooth root canal treatment was advised.

An intra-oral periapical radiograph was taken (Fig. 1) and showed a fractured left central incisor with a wide root canal and an open apex due to incomplete root formation. Dark radiolucency was seen around the apex with breach in continuity of the lamina dura. The radiolucency was surrounded by a wide sclerotic radio opaque band. The right central incisor was showing slight widening of the PDL space in the apical area but the lamina dura was intact. In spite of repeated recommendations for root canal treatment the patient insisted on extraction and replacement of the central incisor.

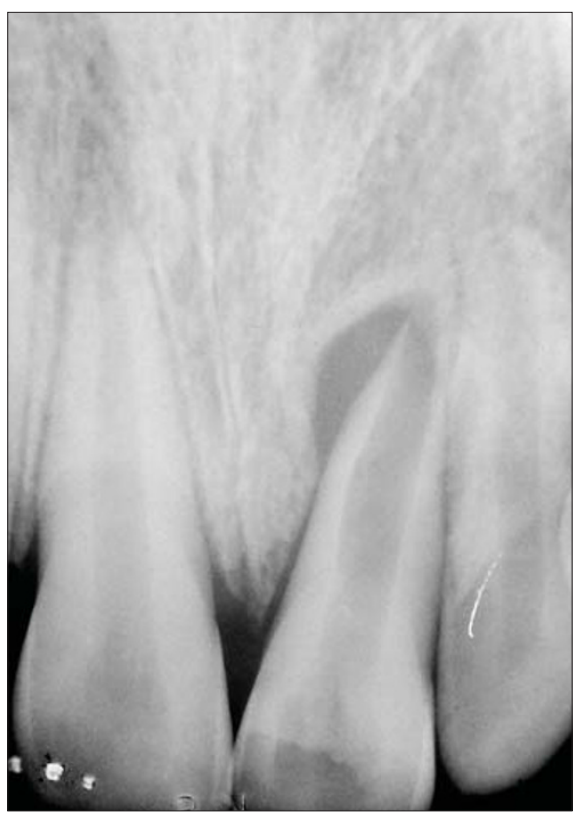

Fig. 1 Intra oral periapical radiograph

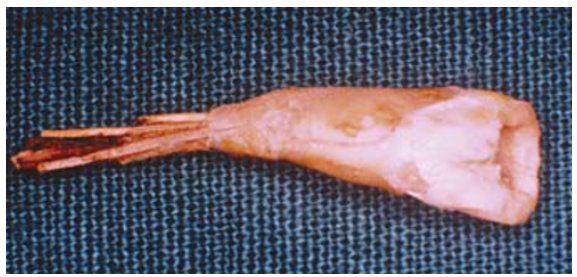

Fig. 2 The tooth with incense sticks

Following infiltration of local anaesthesia (2\% lignocaine with 1:80,000 adrenaline) the tooth was extracted and the peri apical area was curetted and closed with sutures.

To my great surprise the tooth came out with a bunch of incense sticks jutting out from the peri-apical area (Fig. 2). The sticks were tightly packed and extending outside. On further probing the patient confessed that he was putting these sticks in the tooth opening and using them as toothpicks to remove the food lodged inside. Since the sticks were made of thin wood they were radiolucent and not visible radiographically. Healing of the site was uneventful after a week. Later there was no subsequent follow up from the patient's end.

This is a very unusual case as it was very difficult to identify this foreign body in the canal radiologically and an attempt to do a root canal treatment would have brought a lot of surprises and even failure.

A lot of cases are reported in the literature regarding foreign bodies in the root canal and peri-apical area but most of them were radiologically visible. In this case it was radiolucent. Balto ${ }^{1}$ reported a case of a metallic pin in the root canal and peri radicular area. Chenail and Teplitsky $^{2}$ viewed lateral and patient placed foreign bodies and listed nails, pencil leads, toothpicks and pins among the list.

Hall $^{3}$ cited the introduction of a wooden object into the pulp chamber of a tooth in a child with vitamin D resistant rickets. Lamster ${ }^{4}$ reported two cases in which pencil lead and a metal needle were found in the root canal and apical area respectively. Cataldo ${ }^{5}$ reported a staple and a portion of paper clip found in the root canal. Walvekar ${ }^{6}$ reported two cases in which a broken sewing needle in one tooth and a broken bur head in another were reported.

These cases stress the limitations of conventional radiography in diagnosing and treating endodontic problems.

\section{R. M. Gadgil, India}

1. Balto H. A radiopaque object in periradicular area. Egypt Dent J 2002; 48: 1023-1026.

2. Chenail B L, Teplitsky P E. Orthograde ultrasonic retrieval of root canal obstruction. J Endod 1987; 13: 186.

3. Hall J B. Endodontics - patient performed. J Dent Child 1969; 36: 213-215.

4. Lamster I B, Barenic J T. Foreign objects in root canal, review of literature and report of two cases. Oral Surg 1977: 44: 483-486.

5. Cataldo E. Unusual foreign objects in pulp chamber. Oral Surg Oral Med Oral Pathol 1976; 42: 851

6. Walvekar S V, Al-Duwairi Y, Al-Kandari A M, AlQuoud 0 A. Unusual foreign objects in the root canal. J Endod 1995; 21: 526-527.

DOI: $10.1038 /$ sj.bdj.2009.862 